\title{
MAXIMUM OF CERTAIN FUNDAMENTAL LAGRANGE INTERPOLATION POLYNOMIALS ${ }^{1}$
}

\author{
M. S. WEBSTER
}

This note extends some of the results obtained in a previous paper ${ }^{2}$ which we shall designate as I. The notations are the same.

We are concerned with the polynomials

$$
l_{k}^{(n)}(x) \equiv \frac{\phi_{n}(x)}{\phi_{n}^{\prime}\left(x_{k}\right)\left(x-x_{k}\right)}, \quad k=1,2, \cdots, n,
$$

where $\phi_{n}(x) \equiv\left(x-x_{1}\right)\left(x-x_{2}\right) \cdots\left(x-x_{n}\right)$ is the Jacobi polynomial of degree $n$ which satisfies the differential equation $\left(1-x^{2}\right) \phi_{n}{ }^{\prime \prime}(x)$ $+[\alpha-\beta-(\alpha+\beta) x] \phi_{n}^{\prime}(x)+n(n+\alpha+\beta-1) \phi_{n}(x)=0$. The parameters $\alpha, \beta$ are positive and $n$ is a positive integer. It is known that $-1<x_{n}<x_{n-1}<\cdots<x_{1}<1$. Throughout the paper, $x$ is always restricted to the interval $-1 \leqq x \leqq 1$.

It was shown in I, for example, that, if $\alpha=\beta=\frac{3}{2}, \max \left|l_{k}^{(n)}(x)\right|<2$ and $l_{1}^{(n)}(1) \rightarrow 2$ as $n \rightarrow \infty$.

Now we use ${ }^{3}$

$$
\phi_{n}(1)=\frac{2^{n} \Gamma(n+\beta) \Gamma(n+\alpha+\beta-1)}{\Gamma(\beta) \Gamma(2 n+\alpha+\beta-1)}
$$

and the asymptotic expressions ${ }^{4}$

$$
\begin{aligned}
\phi_{n}(\cos \theta)= & \frac{2^{n} \Gamma(n+1) \Gamma(n+\alpha+\beta-1)}{(\pi n)^{1 / 2} \Gamma(2 n+\alpha+\beta-1)}\left(\sin \frac{\theta}{2}\right)^{1 / 2-\beta}\left(\cos \frac{\theta}{2}\right)^{1 / 2-\alpha} \\
& \cdot\left\{\cos [N \theta-(2 \beta-1) \pi / 4]+(n \sin \theta)^{-1} O(1)\right\}, \\
\phi_{n}(\cos \theta)= & \frac{2^{n} \Gamma(n+1) \Gamma(n+\alpha+\beta-1)}{\Gamma(2 n+\alpha+\beta-1)}\left(\sin \frac{\theta}{2}\right)^{1-\beta}\left(\cos \frac{\theta}{2}\right)^{1-\alpha} \\
& \cdot\left\{\frac{\Gamma(n+\beta)}{\Gamma(n+1)}\left(\frac{\theta}{\sin \theta}\right)^{1 / 2} \frac{J_{\beta-1}(N \theta)}{N^{\beta-1}}+\theta^{1 / 2} O\left(n^{-3 / 2}\right)\right\},
\end{aligned}
$$

where $N=n+(\alpha+\beta-1) / 2, c n^{-1} \leqq \theta \leqq \pi-\epsilon, c, \epsilon$ positive constants and

1 Presented to the Society, April 13, 1940.

${ }^{2} \mathrm{M}$. Webster, Note on certain Lagrange interpolation polynomials, this Bulletin, vol. 45 (1939), pp. 870-873.

${ }^{3} \mathrm{C}$. Winston, On mechanical quadratures formulae involving the classical orthogonal polynomials, Annals of Mathematics, (2), vol. 35 (1934), pp. 658-677.

${ }^{4}$ G. Szegö, Orthogonal Polynomials, American Mathematical Society Colloquium Publications, vol. 23, 1939, pp. 191-192, 121, 123. 
where $J_{m}(x)$ is Bessel's function of order $m$. Since $\phi_{n}^{\prime}(x ; \alpha, \beta)$ $=n \phi_{n-1}(x ; \alpha+1, \beta+1)$, these yield immediately the following results:

LEMma. For $x_{k}$ such that $-1+\epsilon \leqq x_{k} \leqq 1-\epsilon$ and $\left|x-x_{k}\right| \geqq \epsilon^{\prime}>0$, $\max \left|l_{k}^{(n)}(x)\right| \rightarrow 0$ as $n \rightarrow \infty$ even if $x \rightarrow \pm 1\left(\alpha, \beta<\frac{3}{2} ; \epsilon, \epsilon^{\prime}>0\right)$.

THeOREM 1. For $x_{k}$ such that $-1+\epsilon \leqq x_{k} \leqq 1-\epsilon$ and $\left|x-x_{k}\right| \geqq \epsilon^{\prime}>0$, $\max \left|l_{k}^{(n)}(x)\right|=O\left(n^{\gamma}\right)$ as $n \rightarrow \infty$ where $\max (\alpha, \beta)=\gamma>\frac{3}{2} ; \epsilon, \epsilon^{\prime}>0$. The exponent $\gamma$ cannot be decreased.

The method used in the proof of Theorem 5 in I really gives the following slightly stronger result:

Theorem 2. If $-1+\epsilon \leqq x_{k} \leqq 1-\epsilon,-1+\epsilon^{\prime} \leqq x \leqq 1-\epsilon^{\prime}, \max \left|l_{k}^{(n)}(x)\right|$ $\rightarrow 1$ as $n \rightarrow \infty\left(\epsilon, \epsilon^{\prime}>0\right)$.

Combining Theorem 2 and the lemma, we obtain the following:

THEOREM 3. For $x_{k}$ such that $-1+\epsilon \leqq x_{k} \leqq 1-\epsilon, \max \left|l_{k}^{(n)}(x)\right| \rightarrow 1$ as $n \rightarrow \infty\left(\alpha, \beta<\frac{3}{2}, \epsilon>0\right)$.

This result is a considerable improvement over Theorem 5 in I. Moreover, if the hypothesis $-1+\epsilon \leqq x_{k} \leqq 1-\epsilon$ is removed, the theorem is not true as Erdös and Grünwald ${ }^{5}$ showed in case $\alpha=\beta=\frac{1}{2}$. In view of Theorems $1,4,5,6$, the restriction $\alpha, \beta<\frac{3}{2}$ is also necessary. In particular, this theorem holds for the case of Tschebycheff $\left(\alpha=\beta=\frac{1}{2}\right)$ and Legendre $(\alpha=\beta=1)$ polynomials.

THeorem 4. If $\alpha=\beta=\frac{3}{2}$ and $x_{k} \rightarrow$ t as $n \rightarrow \infty$, then $\max \left|l_{k}^{(n)}(x)\right| \rightarrow 1$ $+|t|$ as $n \rightarrow \infty(-1 \leqq t \leqq 1)$. This is also an upper bound if $\left|x_{k}\right|<|t|$ at least for large values of $n$.

Proof. It was shown in I that $l_{k}^{(n)}(1)=1+x_{k}$ and for $x_{k+1} \leqq x \leqq x_{k-1}$, $\max \left|l_{k}^{(n)}(x)\right|<1.87$. Since (I) $\max \left|l\left({ }_{k}^{(n)} x\right)\right|$ is attained either between $x_{k+1}$ and $x_{k-1}$ or at $x= \pm 1$, the theorem is valid for $t=1$ and, by symmetry, for $t=-1$.

If $|t|<1$, the preceding paragraph and Theorem 2 complete the proof. In fact, $\max \left|l_{k}^{(n)}(x)\right|=1+\left|x_{k}\right|$ at least for large $n$.

The next two theorems are obtained in a similar manner.

THEOREM 5. If $\alpha=\frac{1}{2}, \beta=\frac{3}{2}$ and $x_{k} \rightarrow t$ as $n \rightarrow \infty$, then $\max \left|l_{k}^{(n)}(x)\right|$ $\rightarrow 4 / \pi$ if $t=-1,1$ if $-1<t \leqq-\frac{1}{2},(2(1+t))^{1 / 2}$ if $-\frac{1}{2} \leqq t \leqq 1$.

THEOREM 6. If $\alpha=\frac{3}{2}, \beta=\frac{1}{2}$ and $x_{k} \rightarrow t$ as $n \rightarrow \infty$, then $\max \left|l_{k}^{(n)}(x)\right|$ $\rightarrow[2(1-t)]^{1 / 2}$ if $-1 \leqq t \leqq \frac{1}{2}, 1$ if $\frac{1}{2} \leqq t<1,4 / \pi$ if $t=1$.

'Erdös and Grünwald, Note on an elementary problem of interpolation, this Bulletin, vol. 44 (1938), pp. 515-518. 


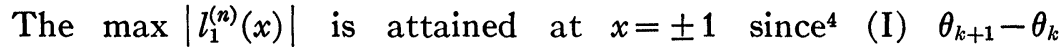
$\leqq 2 \pi /(2 n+\alpha+\beta-1)$ provided $\frac{1}{2} \leqq \alpha, \beta \leqq \frac{3}{2}$ and $x_{k} \equiv \cos \theta_{k}$. Using the second asymptotic formula and the fact that $n \theta_{k} \rightarrow j_{k}$ as $n \rightarrow \infty$ where $j_{k}$ is the $k$ th positive zero of $J_{\beta-1}(x)$, we find that

$$
\left|l_{k}^{(n)}(1)\right| \rightarrow\left(\frac{1}{2} j_{k}\right)^{\beta-2}\left|\Gamma(\beta) J_{\beta}\left(j_{k}\right)\right|^{-1} \quad \text { as } \mathrm{n} \rightarrow \infty, k \text { constant, }
$$

$l_{1}^{(n)}(-1) \rightarrow 0$ which proves the theorem:

Theorem 7. Max $\left|l_{1}^{(n)}(x)\right| \rightarrow\left(\frac{1}{2} j_{1}\right)^{\beta-2}\left|\Gamma(\beta) J_{\beta}\left(j_{1}\right)\right|^{-1}$ as $n \rightarrow \infty$ (where $\frac{1}{2} \leqq \alpha, \beta \leqq \frac{3}{2}, j_{1}$ is first positive zero of $\left.J_{\beta-1}(x)\right)$.

A similar result holds for $l_{n}^{(n)}(x)$ if $\beta$ is replaced by $\alpha$.

For Legendre polynomials $(\alpha=\beta=1)$ this limit is approximately 1.602. For $\alpha=\beta=\frac{1}{2}$ and $\alpha=\beta=\frac{3}{2}$ the limit of Theorem 7 is also an upper bound for $\max \left|l_{1}^{(n)}(x)\right|$ and $\max \left|l_{k}^{(n)}(x)\right|$. Whether this is true, in general, remains unanswered.

Purdue University

\section{AN INVARIANCE THEOREM FOR SUBSETS OF $S^{n 1}$}

\section{SAMUEL EILENBERG}

The purpose of this paper is to establish the following.

INVARIANCE THEOREM. Let $A$ and $B$ be two homeomorphic subsets of the $n$-sphere $S^{n}$. If the number of components of $S^{n}-A$ is finite, then it is equal to the number of components of $S^{n}-B$.

In the case when $A$ and $B$ are closed this theorem is a very well known consequence of Alexander's duality theorem and its generalizations. In our case we also derive our result as a consequence of a duality theorem. However, the duality is established only for the dimension $n-1$.

Given a metric space $X$ we shall say that $\Gamma^{k}$ is a $k$-cycle in $X$ if there is a compact subset $A$ of $X$ such that $\Gamma^{k}$ is a $k$-dimensional convergent (Vietoris) cycle in $A$ with coefficients modulo 2 . We shall write $\Gamma^{k} \sim 0$ if $\Gamma^{k} \sim 0$ holds in some compact subset of $X$. The homology group of $X$ obtained this way will be denoted by $\mathfrak{F}^{k}(X)$; the corresponding connectivity number, by $p^{k}(X)$. The number $p^{k}(X)$ can be either finite or $\infty$.

\footnotetext{
1 Presented to the Society, December 28, 1939.
} 